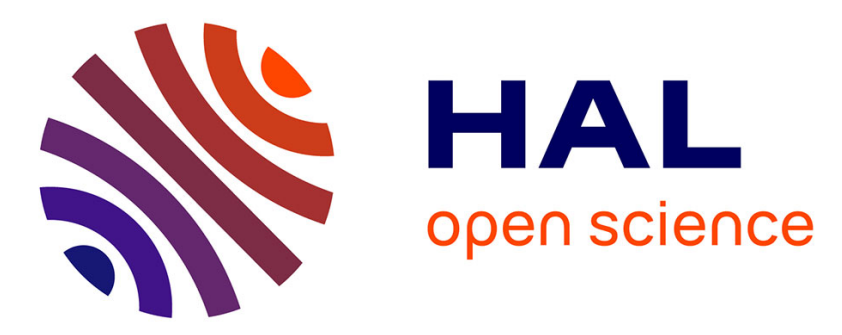

\title{
Qualitative Bipolar Decision Frameworks Viewed as Pessimistic/Optimistic Utilities
}

Florence Dupin de Saint Cyr - Bannay, Romain Guillaume

\section{To cite this version:}

Florence Dupin de Saint Cyr - Bannay, Romain Guillaume. Qualitative Bipolar Decision Frameworks Viewed as Pessimistic/Optimistic Utilities. 30th IEEE International Conference on Fuzzy Systems (FUZZ-IEEE 2021), IEEE, Jul 2021, Luxembourg, Luxembourg. pp.1-14, 10.1109/FUZZ45933.2021.9494517 . hal-03327992

\section{HAL Id: hal-03327992 \\ https://hal.science/hal-03327992}

Submitted on 27 Aug 2021

HAL is a multi-disciplinary open access archive for the deposit and dissemination of scientific research documents, whether they are published or not. The documents may come from teaching and research institutions in France or abroad, or from public or private research centers.
L'archive ouverte pluridisciplinaire HAL, est destinée au dépôt et à la diffusion de documents scientifiques de niveau recherche, publiés ou non, émanant des établissements d'enseignement et de recherche français ou étrangers, des laboratoires publics ou privés.

\section{(c)(1)}

Distributed under a Creative Commons Attribution| 4.0 International License 


\title{
Qualitative Bipolar Decision Frameworks Viewed as Pessimistic/Optimistic Utilities*
}

\author{
Florence Dupin de Saint-Cyr Romain Guillaume \\ Université de Toulouse-IRIT \\ Toulouse, France \\ florence.bannay@irit.fr_ｒomain.guillaume@irit.fr
}

August 27, 2021

\begin{abstract}
A bipolar structure called BLF expresses knowledge about decisions in terms of decision principles that are ranked and polarized according to the utility of the consequences of these decisions. A BLF allows us to compare decisions under incomplete knowledge. For a given decision, the BLF returns a vector of utility/dis-utility in terms of achievement of positive/negative goals. Decisions are compared thanks to these vectors. In this paper we focus on the link between the uncertain knowledge aggregation made by the BLF and classical aggregation functions used in decision under uncertainty and multi-criteria approaches. The main benefit of a BLF is that thanks to the bipolar scale, positive and negative goals can be dealt with independently under their own point of view (each of them being either pessimistic or optimistic).
\end{abstract}

\section{Introduction}

Decision is one of the most challenging rational act in everyday life, indeed people are always trying to find the best decision to make in professional situations as well as in the private sphere. However, making a good decision, even an excellent one, is not enough, since the main issue is to convince the actors (including possibly oneself) that it is the right thing to do. In order to convince people, it is necessary to be able to accompany this decision with explanations justifying that this choice takes into account criteria which have been previously well

${ }^{*}$ This is a draft version, the paper has been accepted in 30th IEEE International Conference on Fuzzy Systems (FUZZ-IEEE 2021), Luxembourg, July 11-14.

Romain Guillaume has benefited from the AI Interdisciplinary Institute ANITI funding. ANITI is funded by the French "Investing for the Future - PIA3" program under the Grant agreement nANR-19-PI3A-0004. 
established and approved. This kind of explanation is not often present in the literature, because many approaches select a decision on the basis of black-box numeric methods that are not easy to interpret.

Decision theory is based on at least three parameters: i) the set of alternatives (or decisions); ii) the evaluation of the possible outcomes: it can be relational or numerical (then it is called utility); iii) the link between decisions and outcomes. In [1], the authors consider three possible epistemic situations about the link between decisions and outcomes: 1) when the link is deterministic: we face a problem of decision under certainty; 2) when each action leads to one set of possible specific outcomes with a known probability associated to each outcome: it is called decision under risk; 3) when an action may lead to outcomes whose probability is completely unknown: it is a decision under uncertainty. In this paper we are interested in decision under uncertainty where this uncertainty is more ordinal than numerical. Namely we use possibility theory to encode the uncertain knowledge about the outcomes. We distinguish two cases of uncertainty: binary uncertainty, called "ignorance", where a world is either completely possible or impossible, and gradual uncertainty where the worlds are more or less certain and their certainty can be compared. Another parameter is the ability to distinguish between good and bad aspects of the outcomes, called bipolarity, see e.g. [2]

In this paper we are going to study the decision selection process that can be done with a Bipolar Layered Framework (BLF). We aim at comparing the results of the BLF to the ones obtained by classical qualitative decision approaches. In this context, we are not considering that numerical utility values are available hence we can not use the Choquet expected utility or maxmin Expected utility but we can use their qualitative counterparts Sugeno and maxmin/maxmax criteria. Maxmin criteria is a pessimistic criterion which focuses on the worst possible utility that can be obtained for a situation resulting from the decision, maxmax is an optimistic one: it compute the best possible utilitity that could be reached by a decision. Besides, when dealing with uncertainty, it is convenient to have both a compact and exception tolerant representation of information. This is why default rules [3] are used to relate alternatives to outcomes in the BLF. The translation of default rules into constraints about the uncertainty levels of worlds was used by [4] in order to relate BLF to qualitative decision theory and also to propose a process to build a BLF from a possibility distribution on alternatives given a utility function on outcomes. Then [5] proposed to use a BLF in order to select the pieces of information to obtain in order to make a better decision. Lastly, [6] have extended the BLF framework in order to express the strength of some arguments and also the possibility to express that some features may strengthen the confidence in some arguments.

There are very few bipolar approaches that are able to handle uncertain knowledge apart from [7] and [8], and few deal with multi-criteria namely [9]. As far as we know, none of them deal with both, i.e., uncertainty and multiplecriteria, we will come back to them in the final discussion. This paper studies the role played by the bipolar setting of the BLF in the decision mechanism: for this purpose we are going to compare the results that can be obtained by 
different bipolar distributions of the goals in the BLF with the results that could be obtained with classical qualitative approaches of decision under ignorance (based on the decision maker optimism).

In this paper, we first give some background about qualitative decision and recall the classical example of Savage. In a second section we present the BLF and illustrate it on several representation variants of Savage's example. Then we introduce the main result of this paper: the BLF is a generalization of the maxmin and maxmax criteria in the case of total ignorance. Thus, this paper establishes that, in addition to 1) its visual aspect, 2) its ability to facilitate the justification of the decision, 3) its clear identification of relevant information, the BLF generalizes the classical approaches of decision making under qualitative uncertainty.

\section{Notations, Background and Savage's Example}

\section{$2.1 \quad$ Notations}

We consider a set $\Omega$ of alternatives (or worlds) on which information is available. This information can be expressed in two languages $\mathscr{L}_{F}$ (a propositional language based on a vocabulary $\mathcal{V}_{F}$ ) for expressing information about some features that are believed to hold for an alternative and $\mathscr{L}_{G}$ (another propositional language based on a distinct vocabulary $\mathcal{V}_{G}$ ) representing information about the achievement of some tangible results (called goals) when an alternative is selected. In the propositional languages used here, the logical connectors or, and, not are denoted respectively by $\vee, \wedge$, and $\neg$. A literal is a propositional symbol $x$ or its negation $\neg x$. Note that we use $\neg l$ to represent the literal which is the negation of $l$, i.e., if $l=x$ then $\neg l=\neg x$ and if $l=\neg x$ then $\neg l=x$. The set of literals of $\mathscr{L}_{G}$ are denoted by $L I T_{G}$. Classical inference, logical equivalence and contradiction are denoted respectively by $=, \equiv, \perp$. We use the symbol $\rightsquigarrow$ as a defeasible entailment symbol: $\varphi \rightsquigarrow g$ is interpreted by when an alternative satisfies $\varphi$ then generally the goal $g$ is achieved by this alternative (but exceptions may arise).

In our framework, decisions are characterized by formulas representing their observable effect. Among the formulas of $\mathscr{L}_{F}$ some formulas (we call $D$ this subset of decision formulas) can be distinguished because the user can act on them. A decision $d \in \mathscr{L}_{F}$ is a way to select a set of alternatives (or worlds) denoted $\Omega^{d}$, we say that $\varphi \in \mathscr{L}_{G} \cup \mathscr{L}_{F}$ is satisfied by the decision $d$, abbreviated $d \models \varphi$, if and only if $\Omega^{d} \models \varphi\left(\varphi\right.$ holds in every world of $\left.\Omega^{d}\right)$.

\subsection{Savage's Omelet[10]}

Example 1 (Savage's Omelet). Someone has just broken five good eggs into a bowl when an agent comes and volunteer to finish making the omelet. The sixth egg lies unbroken beside the bowl. For some reason it must either be used for the omelet or wasted altogether. The agent must decide what to do with this 
unbroken egg. The agent must decide among three acts only. Namely:

- bi: to break an egg to join the other five eggs,

- bb: to break an egg into a saucer for inspection,

- ta: to throw an egg away without inspection.

In this example, the features for describing the problem are $\mathcal{V}_{F}=\left\{e_{6}, s t, n b\right\}$, representing respectively "to have a good sixth egg", "there is a shell on the table" (meaning that the 6th egg has been broken by the agent, ᄀst means that the egg was thrown away directly to the garbage), "a new bowl is on the table", yielding to eight possible situations. The three main issues at stake in this problem are: ensure to have an omelet with a maximum number of eggs and avoid to waste too many eggs (minimize the number of squandered eggs), avoid extra-dishes. The values associated to these issues are determined by the situation, for instance in situation $\omega_{3}$ the sixth egg is a bad egg, there is no new bowl on the table hence the decision maker has chosen to brake it inside the bowl hence the omelet is wasted, giving an omelet size of 0, five eggs are squandered, and there is no extra-dishes (see Table 1). The acts are linked with the features as follows: bi causes st $\wedge \neg n b$; bb causes st $\wedge n b$ and ta causes $\neg$ st.

\begin{tabular}{|l|l|l|l||ll|l|}
\hline \multirow{2}{*}{ worlds } & \multicolumn{3}{|c||}{ description } & \multicolumn{3}{c|}{ goals } \\
\cline { 2 - 7 } & $\begin{array}{l}\text { good } \\
6 \text { th egg } \\
e_{6}\end{array}$ & $\begin{array}{l}\text { shell on } \\
\text { table } s t\end{array}$ & $\begin{array}{l}\text { new } \\
\text { bowl } \\
n b\end{array}$ & $\begin{array}{l}\text { omelet } \\
\text { size }\end{array}$ & $\begin{array}{l}\text { squan } \\
\text { dered }\end{array}$ & $\begin{array}{l}\text { extra- } \\
\text { dishes }\end{array}$ \\
\hline$\omega_{1}$ & 0 & 0 & 0 & 5 & $0^{*}$ & 0 \\
\hline$\omega_{2}$ & 0 & 0 & 1 & 5 & $0^{*}$ & 1 \\
\hline$\omega_{3}$ & 0 & 1 & 0 & 0 & 5 & 0 \\
\hline$\omega_{4}$ & 0 & 1 & 1 & 5 & $0^{*}$ & 1 \\
\hline$\omega_{5}$ & 1 & 0 & 0 & 5 & 1 & 0 \\
\hline$\omega_{6}$ & 1 & 0 & 1 & 5 & 1 & 1 \\
\hline$\omega_{7}$ & 1 & 1 & 0 & 6 & 0 & 0 \\
\hline$\omega_{8}$ & 1 & 1 & 1 & 6 & 0 & 1 \\
\hline
\end{tabular}

Table 1: The eight situations with their realized goals ${ }^{1}$.

In our framework, the three quantitative criteria can be represented by binary propositional variables in $\mathscr{L}_{G}$ : at least one (respectively five) egg(s) is squandered, abbreviated $\boldsymbol{s} \mathbf{1}$ (respectively s5); an omelet of size of at least 5 (respectively 6) eggs is obtained, abbreviated o5 (respectively o6), extra-dishes is produced, abbreviated ed. These issues are called "goals", $\mathcal{V}_{G}=\left\{s_{1}, s_{5}, o_{5}, o_{6}, e d\right\}$, they have different importance, this can be expressed by a utility function u. Here we consider: $u\left(s_{5}\right)<u\left(s_{1}\right)<u(e d)<u\left(o_{5}\right)<u\left(o_{6}\right)$. These utilities induce the following strict preference ordering between worlds (according to the goals they achieve shown in Table 1): $\omega_{3}<\omega_{6}<\omega_{5}<\omega_{2}=\omega_{4}<\omega_{1}<\omega_{8}<\omega_{7} .{ }^{2}$

\footnotetext{
${ }^{1} \mathrm{~A}$ bad egg that has been thrown away is not considered as squandered.

${ }^{2}$ Note that $\omega_{2}$ and $\omega_{6}$ are situations where the sixth egg has been thrown away but a new bowl is on the table (these situations cannot occur when we consider only the three actions $b b, b i$ and $t a)$.
} 


\subsection{Decision criteria under ignorance}

In the context of decision under ignorance, the knowledge about the world is drastic in the sense that a world is either known to be possible or known to be impossible. In other words making a decision $d$ leads to obtain a set of selected worlds that are completely possible while the other ones are considered impossible to be achieved by the decision $d$. Moreover, we dispose of a utility function on the set of worlds $u: \Omega \rightarrow \mathbb{R}$ which is given.

Hence, the choice of the best decision $d$ depends on the extreme evaluations of the worlds achieved by that decision $d$ : [11] defines the minmax preference as follows:

Definition 1 (minmax preference). Given two decisions $d_{1}$ and $d_{2}, d_{1}$ is min$\max$ preferred to $d_{2}$ if

$$
\left\{\begin{array}{l}
\min _{\omega \in \Omega^{d_{1}}} u(\omega) \geq \min _{\omega \in \Omega^{d_{2}}} u(\omega) \text { and } \\
\max _{\omega \in \Omega^{d_{1}}} u(\omega) \geq \max _{\omega \in \Omega^{d_{2}}} u(\omega)
\end{array}\right.
$$

Example 2. bi (which leads to $\omega_{3}$ and $\omega_{7}$ ) is minmax incomparable with bb (leading to $\omega_{4}$ and $\omega_{8}$ ) and with ta (leading to $\omega_{1}$ and $\omega_{5}$ ). bb is minmax preferred to ta since the max utility for bb is to get all the goals achieved except ᄀed (in $\omega_{8}$ ) which is better than the best world reachable with ta, also the worse utility for bb is to reach $\omega_{4}$ which does not squandered any egg while the worst world for ta $\left(\omega_{5}\right)$ squanders a good egg.

Another approach is to focus on one of these extreme evaluations in order to choose the best decision. Two criteria have been proposed one based on minimal possible utility and one on the maximal possible utility, namely the maxmin and maxmax criteria. maxmin is a pessimistic criterion since it focuses on the worst possible utility that can be obtained with a decision and maxmax is an optimistic criterion since it focuses on the best possible utility reachable with a decision.

Definition 2 (pessimistic and optimistic criterion).

The best pessimistic decision value is $\max _{d \in \mathbf{D}} \min _{\omega \in \Omega^{d}} u(\omega)$

The best optimistic decision value is $\max _{d \in \mathbf{D}} \max _{\omega \in \Omega^{d}} u(\omega)$

In other words, $d_{1}$ is pessimistically preferred to $d_{2}$ if $\min _{\omega \in \Omega^{d_{1}}} u(\omega) \geq$ $\min _{\omega \in \Omega^{d_{2}}} u(\omega)$ and it is optimistically preferred if $\max _{\omega \in \Omega^{d_{1}}} u(\omega) \geq \max _{\omega \in \Omega^{d_{2}}} u(\omega)$.

Example 3. The best pessimistic decision is bb (which leads to $\omega_{4}$ at worst) and the best optimistic decision is bi (which leads to $\omega_{7}$ at best).

Pessimistic and optimistic approaches have extreme behaviors. Recently a criterion which generalizes it by allowing for taking into account the optimism of the decision maker in a qualitative context have been proposed in [12]. This criterion is the $R_{*}^{e}$ criteria where $e \in[-1,1]$ models the optimism of the decision maker, it is a threshold under which the decision maker will consider that the worst utility and over which she will assume to obtain the best one. 
Definition 3 ( $R_{*}^{e}$-criterion). The best $R_{*}^{e}$ decision value is: $\max _{d \in \mathbf{D}}\left\{\begin{array}{l}\min _{\omega \in \Omega^{d}} u(\omega) \text { if } \min _{\omega \in \Omega^{d}} u(\omega)<e \\ \max _{\omega \in \Omega^{d}} u(\omega) \text { otherwise }\end{array}\right.$

Example 4. If the threshold of optimism is that the decision is good as soon as no more than one egg is squandered: $e=u\left(s_{1}\right)$ then $b b$ is $R_{*}^{e}$-preferred to ta and bi.

\section{Bipolar Layered Framework}

\subsection{Definition}

A Bipolar Layered Framework (BLF) is a visual structure [4] that represents all explicit information known about a decision domain in a compact way. Hence, it contains both the knowledge for reasoning about the achievement of goals and the preference information associated to these goals: namely their polarities and their importance level. The polarity of a goal is positive if it is a desirable result, negative when this result should be avoided. In order to compare this approach to classical ones we rephrase the definition of [4]:

Definition 4 (BLF). Given a utility function $u$ : $\left.L I T_{G} \rightarrow\right]-1,1[$.

$A$ BLF is a tuple $(G, \mathcal{P}, \mathcal{I}, l)$.

- $G \subseteq L I T_{G}$ is a set of literals goals s.t. $\forall g \in G, u(g) \neq 0 . G$ is separated into $G^{\oplus}$ and $G^{\ominus}$ where $G^{\oplus}=\{g \in G \mid u(g)>0\}$ and $G^{\ominus}=\{g \in G \mid u(g)<$ $0\}$.

- $\mathcal{P}$ is a set of decision principles (DPs). A decision principle is an expression $\varphi \rightsquigarrow g$ where $\varphi \in \mathscr{L}_{F}, g \in L I T_{G}$ which expresses that there is a causal link between $\varphi$ and $g$ of the form generally when $\varphi$ holds $g$ is realized

- $\mathcal{I}$ is a set of inhibitors. An inhibitor is a pair $(\psi, \varphi \rightsquigarrow g)$ where $\psi \in \mathscr{L}_{F}$ and $\varphi \rightsquigarrow g \in \mathcal{P}$ which expresses that when $\psi$ holds the causal link between $\varphi$ and $g$ is broken

- $l: G \rightarrow[0,1]$ represents the level of importance of each goal of the BLF, it is defined by $\forall g \in G^{\oplus}, l(g)=u(g)$ and $\forall g \in G^{\ominus}, l(g)=-u(g)$.

Note that a BLF is a compact representation of a possibility distribution $\pi$ and a utility function $u$ on worlds (see [4] where a process for generating automatically the BLF from $\pi$ and $u$ is explained). A BLF is associated to a visual representation as defined below (an example is shown in Fig 1).

Definition 5 (BLF Visual representation). A BLF $(G, \mathcal{P}, \mathcal{I}, l)$ is associated with a tripartite layered graph where the arcs are defined by $\mathcal{I}$ and the vertices are separated in three sets: $\ominus=\left\{\varphi \rightsquigarrow g \in \mathcal{P} \mid g \in G^{\ominus}\right\}$, the set of DPs with a negative achievement; $\oplus=\left\{\varphi \rightsquigarrow g \in \mathcal{P} \mid g \in G^{\oplus}\right\}$, the set of DPs with a positive achievement; Inhib $=\left\{\psi \in \mathscr{L}_{G} \mid(\psi, p) \in \mathcal{I}\right\}$, the set of formulas inhibiting some $D P$ in $\mathcal{P}$.

The vertices of $\ominus$ and $\oplus$ are associated with the level $l$ of their goals. The vertices of Inhib are not layered. 


\subsection{Reasoning with a BLF}

It amounts to take into account the argumentation graph (coming from the visual representation of the BLF) that concerns each goal in order to check whether this goal is realized in the current context $K \subset \mathscr{L}_{F}$. The goals $\left(\in \mathscr{L}_{G}\right)$ that are realized are the conclusions of the uninhibited DPs in the BLF given that $K$ holds:

Definition 6 (realized goals). Given a $B L F B=(G, \mathcal{P}, \mathcal{I}, l)$, a goal g in $L_{I} T_{G}$ is realized wrt $B$ and $K \in \mathscr{L}_{F}$ if there is one $D P p$ in $\mathcal{P}$ s.t. $K \models p$ and $p$ concludes $g$ and $p$ is not inhibited when knowing only $K$.

\subsection{Deciding with a BLF}

One can define many ways to take into account the positive and negative realized goals in order to compare decision. In [13], the authors consider that decisions are supported by positive and negative abstract arguments and they proposed three bipolar qualitative decision rules in order to compare decisions: namely Pareto dominance, bipolar possibility preference and bipolar leximin preference. In this article, we only present the two first ones, they are based on the order of magnitude of a decision $d$ which is defined by a pair composed of a utility (the highest utility of its realized positive goals) and a dis-utility (the lowest utility of its realized negative goals).

Definition 7 (utility, disutility of a decision). Let $d \in \mathscr{L}_{F}$ be a decision and $B=$ $(G, \mathcal{P}, \mathcal{I}, l)$ a $B L F$, the BLF Pareto-evaluation of d is a pair $\left(\right.$ disut $\left._{B}(d), u t_{B}(d)\right)$ s.t.:

$$
\begin{gathered}
\left.\operatorname{disut}_{B}(d)=\max _{g \in G} \ominus(l(g)) \mid g \text { is realized wrt } B \text { and } d\right) \\
u t_{B}(d)=\max _{g \in G \oplus}(l(g) \mid g \text { is realized wrt } B \text { and } d)
\end{gathered}
$$

Pareto dominance decision rule The Pareto dominance ([13]) of a decision $d_{1}$ over a decision $d_{2}$ occurs when the best positive argument in favor of $d_{1}$ is better than the one of $d_{2}$ and the worst negative argument supporting $d_{1}$ is less worse than the worst one supporting $d_{2}$. In order to compare two decisions $d_{1}$ and $d_{2}$ we consider the list of realized goals in each situation wrt the BLF.

Definition 8 (Pareto dominance). Given a $B L F B=(G, \mathcal{P}, \mathcal{I}, l), d_{1}$ and $d_{2}$ in $\mathscr{L}_{F}$,

$$
d_{1} \text { is B-Pareto-preferred to } d_{2} \text { if }\left\{\begin{array}{l}
u t_{B}\left(d_{1}\right) \geq u t_{B}\left(d_{2}\right) \text { and } \\
\operatorname{disut~}_{B}\left(d_{1}\right) \leq \operatorname{disut}_{B}\left(d_{2}\right)
\end{array}\right.
$$

Bipolar possibility preference rule The bipolar possibility preference ([13]) occurs when the most important argument among all the positive arguments in favor of $d_{1}$ and negative arguments in favor of $d_{2}$, is more important than the most important argument among positive arguments for $d_{2}$ and negative arguments for $d_{1}$. 
Definition 9 (biposs preference). Given a $B L F B=(G, \mathcal{P}, \mathcal{I}, l), d_{1}$ and $d_{2}$ in $\mathscr{L}_{F}$,

$$
\begin{gathered}
d_{1} \text { is B-biposs-preferred to } d_{2} \text { if } \\
\max \left(u t_{B}\left(d_{1}\right), \text { disut }_{B}\left(d_{2}\right)\right) \geq \max \left(u t_{B}\left(d_{2}\right), \text { disut }_{B}\left(d_{1}\right)\right)
\end{gathered}
$$

\subsection{Savage's example viewed in the BLF decision context}

All the knowledge contained in Table 1 together with the levels of importance of the goals can be described more compactly by the BLF $B$ of Figure 1 . Here the negative goals are $G^{\ominus}=\left\{s_{1}, s_{5}, e d\right\}$, the positive goals are $G^{\oplus}=\left\{o_{5}, o_{6}\right\}$. Formally $B=\{G, \mathcal{P}, \mathcal{I}, l\}$ where $G=G^{\oplus} \cup G^{\ominus}, \mathcal{P}=\left\{p_{1}, \ldots, p_{6}\right\}, \mathcal{I}=\left\{\left(\neg e_{6}, p_{2}\right)\right.$, $\left.\left(\neg e_{6}, p_{6}\right),\left(\neg n b, p_{3}\right)\right\}$ and $l$ is s.t. $l\left(p_{1}\right)<l\left(p_{2}\right)<l\left(p_{3}\right)=l\left(p_{4}\right)<l\left(p_{5}\right)<l\left(p_{6}\right)$. On the right of Fig. 1 are the DPs with positive goals ordered by importance from bottom to top, on the left are the DPs with negative goals ordered by importance from bottom to top. In this example, we have two inhibitors: $\neg e_{6}$ and $\neg n b$.

The BLF depends on the beliefs of the decision maker, in Savage's example the beliefs about the sixth egg play an important role. Fig. 1 shows the BLF when the decision maker believes that $e_{6}$ is more plausible than $\neg e_{6}$, Fig. 2 contains the BLF corresponding to the opposite belief, in Fig. 3 we show the case where $e_{6}$ is as plausible as its negation. In Fig. 4 we propose to take into account the fact that not getting at least an omelet with five eggs is the worst possible situation, this is done by adding the negative goal $\neg O_{5}$ with the highest level of importance.

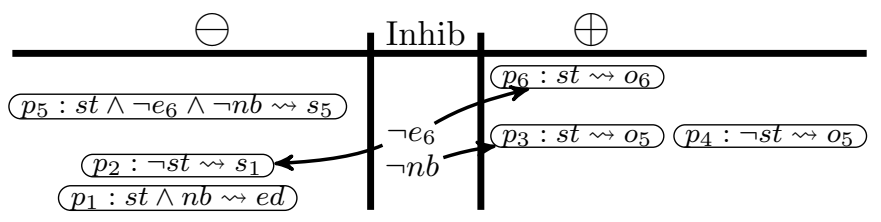

Figure 1: BLF $B 1$ given that $e_{6}$ is more plausible than $\neg e_{6}$

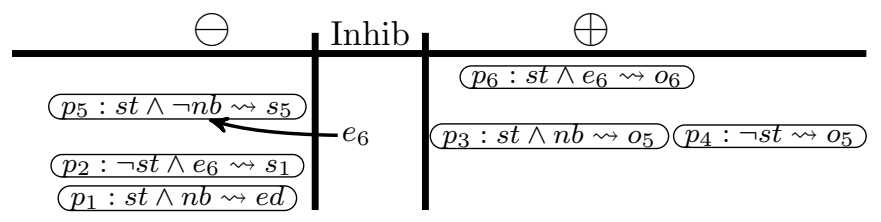

Figure 2: BLF $B 2$ given that $\neg e_{6}$ is more plausible than $e_{6}$

$\mathrm{B} 1$ and B2 are translating two different situations wrt the default beliefs about the sixth egg, an interesting point to notice concerns the DP $p_{3}$. In the 
$\mathrm{BLF} \mathrm{B} 1, p_{3}$ says that if there is shell on the table $(s t)$ then generally we are sure to get an omelet of size $5\left(o_{5}\right)$, unless a new bowl is not used $(\neg n b)$ which would inhibit $p_{3}$ and will make the realization of $o_{5}$ nomore guaranteed. In B2 $p_{3}$ has been changed into the DP stating that generally when there is shell and a new bowl on the table then the goal $o_{5}$ is achieved, with no exception (no inhibitor). In B1 when nothing is known about the new bowl, we will think that $o_{5}$ is achieved, because there is a greater plausibility that the sixth egg is good hence even if we do not use a new bowl the omelet will have a size of at least 5. In B2, in order to be sure that we get an omelet of size at least 5 , we have to know that a new bowl has been used because the sixth egg is more likely to be bad.

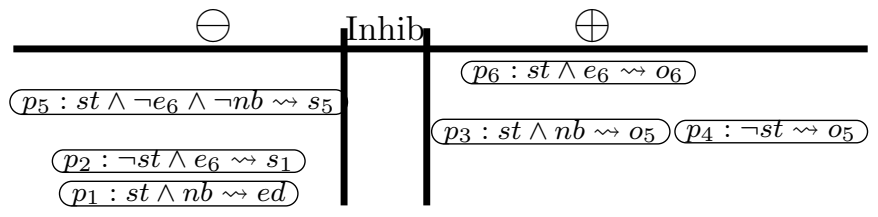

Figure 3: BLF $B 3$ given that $e_{6}$ is as plausible as $\neg e_{6}$

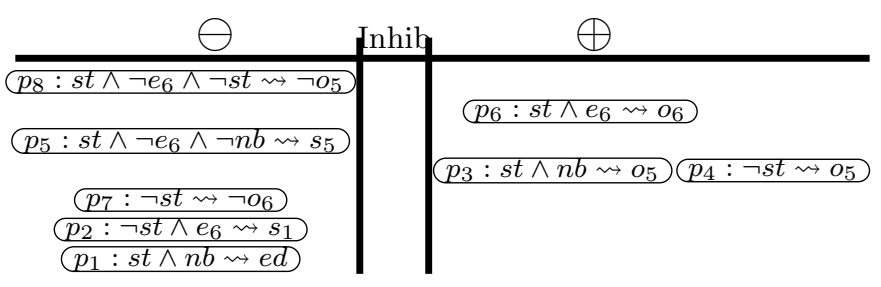

Figure 4: $B 4: \neg O_{5}$ worst negative goal $\left(e_{6}\right.$ as plaus. as $\left.\neg e_{6}\right)$

Let us show how to reason with the four different BLFs. First we can compute the realized goals for the different BLFs given the action (see Definition $6)$. For instance, in B1 when we know that action $b i$ has been performed then we know $K=\{s t \wedge \neg n b\}$, only the DP $p_{6}$ is fired and non inhibited in the context $K$, yielding $o_{6}$. With action $b b$, the context becomes $K=\{s t \wedge n b\}$, then $p_{6}, p_{3}$ and $p_{1}$ are fired and non inhibited yielding to the achievement of the goals $\left\{o_{6}, o_{5}, e d\right\}$. Note that all the DPs or Inhibitors containing $e_{6}$ cannot be fired because we do not know if the sixth egg is good or bad ( $K$ does not contain any information about $e_{6}$ ). All the goals considered to be realized by the different actions according to the four BLFs are described in Table 2.

Once the realized goals are known for a decision $d$, we are in position to compute $u t(d)$ and disut $(d)$ in each BLF according to Definition 7 . Thanks to Definitions 8 and 9, in the BLF B1 (where the sixth egg is more likely to be good) we obtain that it is better wrt Pareto-dominance to break the egg inside the 


\begin{tabular}{lcccc}
\hline Decision & B1 & B2 & B3 & B4 \\
\hline bi (causes $s t \wedge \neg n b)$ & $\left\{o_{6}\right\}$ & $\left\{s_{5}\right\}$ & $\varnothing$ & $\varnothing$ \\
\hline bb (causes $s t \wedge n b)$ & $\left\{o_{6}, o_{5}, e d\right\}$ & $\left\{o_{5}, e d\right\}$ & $\left\{o_{5}, e d\right\}$ & $\left\{o_{5}, e d\right\}$ \\
\hline ta $($ causes $\neg s t)$ & $\left\{s_{1}, o_{5}\right\}$ & $\left\{o_{5}\right\}$ & $\left\{o_{5}\right\}$ & $\left\{\neg O_{6}, o_{5}\right\}$ \\
\hline
\end{tabular}

Table 2: Realized goals for decision $b i, b b$ and $t a$ in each BLF

bowl, indeed $b i$ is Pareto-preferred to $b b$ and $t a$. However, $b i$ is biposs-equivalent to $b b$ and biposs-preferred to $t a$ since the disutility of the extra-dish is neglected in front of the utility of achieving $o_{6}$. In the BLF B2 (where the sixth egg is more plausibly bad) as well as in B3 (where we do not have any information about the sixth egg) the best decision for Pareto-dominance is to throw it away directly $t a$. In B2 and B3, $t a$ is biposs-equivalent to $b b$ and preferred to $b i$. In B4 (where not having an omelet is a negative goal) $b b$ is preferred to $t a$ with regard to Pareto-dominance and incomparable with $b i$ while $b b$ is the Bi-poss most preferred decision.

This example shows that the way the decision maker expresses its goals has a great influence on the results. Indeed, we may consider that a positive goal $g$ can be expressed by $g \in G^{\oplus}$ and be associated with a positive utility $u(g)>0$. But for this same goal $g$ a designer may be more interested to consider that it is important not to achieve $\neg g$ hence he can define $g^{\prime}=\neg g \in G^{\ominus}$ and associate with $g^{\prime}$ a negative utility $u\left(g^{\prime}\right)=u(g)-1$. A third choice is to express both the fact that achieving $g$ is a positive thing and that avoiding $\neg g$ is also important (as it was done with $o_{5}$ ). In other words, either the agent wishes $g$ or the agent wants to avoid $\neg g$ or both.

\section{Deciding under ignorance and fuzzy-BLF}

\subsection{Fuzzy-BLF}

In order to compare BLF with classical decision under uncertainty. We first introduce the notion of fuzzy BLF which imposes the restriction that the positive

and negative goals should be nested and that a more important goal subsumes a less important one.

Definition 10 (Fuzzy BLF). A BLF $B=(G, \mathcal{P}, \mathcal{I}, l)$ is a fuzzy BLF if $\left\{\forall g, g^{\prime} \in G^{\oplus}, \quad\right.$ if $l(g) \geq l\left(g^{\prime}\right)$ then $g \models g^{\prime}$ and $\left\{\forall g, g^{\prime} \in G^{\ominus}, \quad\right.$ if $l(g) \geq l\left(g^{\prime}\right)$ then $\quad g \models g^{\prime}$

Remark 1. Any BLF can be transformed into a Fuzzy BLF by transforming each goal in a lower level to the disjunction of the goals of the higher levels, the two BLFs are equivalent wrt decision making when the original BLF has each positive (resp. negative) goal on a distinct level (as it is the case in our examples). 


\subsection{Bipolarity and optimism of the decision maker}

We show that the aggregation on the positive/negative scale of a BLF is the minmax function over the possible worlds. In other words, the BLF is pessimistic about achieving the goals.

Proposition 1. Given a fuzzy-BLF $B$ and a decision $d \in \mathscr{L}_{F}$, it holds that ${ }^{3}$

$$
u t_{B}(d)=\min _{\omega \in \Omega^{d}} u t(\omega) \quad \operatorname{disut}_{B}(d)=\min _{\omega \in \Omega^{d}} \operatorname{disut}(\omega)
$$

The proofs of Prop.1 is based on a series of Lemmas that we detail below.

Lemma 1. Given a fuzzy-BLF $B=(G, \mathcal{P}, \mathcal{I}, l)$ and a decision $d \in \mathscr{L}_{F}$, if $\min _{\omega \in \Omega^{d}}$ ut $(\omega)>0$ then there is a goal $g \in G^{\oplus}$ such that $g$ is realized wrt $(B$ and $d$ ) and $l(g)=\min _{\omega \in \Omega^{d}} u t(\omega)$.

Proof. Let $\omega^{*}$ be a world with minimum utility among the worlds in $\Omega^{d}: \min _{\omega \in \Omega^{d}} u t(\omega)=$ $u t\left(\omega^{\star}\right)$. Due to Def. 7 , if $u t\left(\omega^{\star}\right)>0$ then $\exists g^{*} \in G^{\oplus}$ such that $\omega^{\star} \models g^{\star}$ and $l\left(g^{*}\right)>0$. The minimality of $\omega^{*}$ implies that for all $\omega \in \Omega^{d}$ there is a goal $g^{\prime}$ s.t. $\omega \models g^{\prime}$ and $l\left(g^{\prime}\right)>l\left(g^{\star}\right)$. Due to Def. $10, g^{\prime} \models g^{\star}$, hence for all $\omega \in \Omega^{d}$, $\omega=g^{\star}$, i.e., $g^{\star}$ is realized wrt $(B$ and $d)$.

Lemma 2. Given a fuzzy-BLF $B$ and a decision $d \in \mathscr{L}_{F}$, there is no goal $g \in G^{\oplus}$ realized wrt to $B$ and $d$ such that $l(g)>\min _{\omega \in \Omega^{d}} u t_{B}(\omega)$.

Proof. Let us suppose that there is a realized goal $g \in G^{\oplus}$ such that $l(g)>$ $\min _{\omega \in \Omega^{d}} u t(\omega)$. If $d \models g$, i.e., any world in $\Omega^{d}$ satisfies $g$. Hence, due to Def. 7 , any world $\omega \in \Omega^{d}$ verifies $u t(\omega) \geq l(g)$ which contradicts $l(g)>\min _{\omega \in \Omega^{d}} u t(\omega)$.

For negative goals, the two similar lemmas also hold.

Lemma 3. Given a decision $d \in \mathscr{L}_{F}$ and a fuzzy-criterion $B L F B=(G, \mathcal{P}, \mathcal{I}, l)$, if $\min _{\omega \in \Omega^{d}} \operatorname{disut}(\omega)>0$ then there is a goal $g \in G^{\ominus}$ such that $g$ is realized wrt $(B$ and $d)$ and $l(g)=\min _{\omega \in \Omega^{d}} \operatorname{disut}(\omega)$

Lemma 4. Given a decision $d \in \mathscr{L}_{F}$ and a fuzzy-BLF $B=(G, \mathcal{P}, \mathcal{I}, l)$, there is no goal $g \in G^{\ominus}$ realized wrt ( $B$ and $d$ ) such that $l(g)>\min _{\omega \in \Omega^{d}} \operatorname{disut}(\omega)$.

Proof. From Lemma 1 and Lemma 2, $\min _{\omega \in \Omega^{d}} u t(\omega)=\max \left(l(g) \mid g \in G^{\oplus}\right.$ is realized wrt $B$ and $d=u t_{B}(d)$ and from Lemma 3 and Lemma $4, \min _{\omega \in \Omega^{d}} \operatorname{disut}(\omega)$ $=\max \left(l(g) \mid g \in G^{\ominus}\right.$ is realized wrt $\left.B_{d}\right)=$ disut $_{B}(d)$; still using the convention $\max _{g \in \varnothing} l(g)=0$.

Proposition 2. Considering a fuzzy-BLF $B=(G, \mathcal{P}, \mathcal{I}, l)$ based on $u$,

- if $G^{\ominus}=\varnothing$, i.e., $G=G^{\oplus}$, then the B-Pareto and the B-Biposs preference orderings on decisions are the same as the maxmin pessimistic criterion relative to $u t_{B}$.

\footnotetext{
${ }^{3}$ With $\min _{\omega \in \varnothing} u t(\omega)=0$ and $\min \omega \in \varnothing \operatorname{disut}(\omega)=0$.
} 
- if $G^{\oplus}=\varnothing$, i.e., $G=G^{\ominus}$, then the B-Pareto and the B-Biposs preference ordering on decisions is the same as the maxmax optimistic criterion relative to $\left(1-\right.$ disut $\left._{B}\right)$.

- if $\forall g \in G^{\oplus}$ then $\neg g \in G^{\ominus}$ and vice versa with $l(g)=l(\neg g)$. then $d_{1}$ is $B$-Pareto-preferred to $d_{2}$ iff $d_{1}$ is minmax preferred to $d_{2}$

Proof. It is enough to note that if $G^{\ominus}=\varnothing$ then, for all $d$ in $\mathscr{L}_{F}$, $\operatorname{disut}_{B}(d)=0$. Hence, the $B$-Pareto preference (Def. 8) and the $B$-biposs preference criteria are using only $u t_{B}(d)=\min _{\omega \in \Omega^{d}} u t(\omega)$ to rank decisions, i.e., $d_{1}$ is $B$-Pareto preferred to $d_{2}$ if $u t_{B}\left(d_{1}\right) \geq u t_{B}\left(d_{2}\right)$, and it is the same for $B$-biposs.

The proof of the second item is similar since in that case the $B$-Pareto preference and the $B$-biposs criteria are using only $\operatorname{disut}_{B}(d)=\min _{\omega \in \Omega^{d}} \operatorname{disut}(\omega)$ $=1-\max _{\omega \in \Omega^{d}}(1-\operatorname{disut}(\omega))$. From the two first point it is easy to check the third item.

Note that, in Proposition 2, the negative case is related to a utility function that is equal to one minus the one used by the BLF, because in the BLF the negative goals (which have a negative utility) are associated to a positive level in $[0,1]$ that translates their (negative) importance, hence we reverse this scale in order to compare our approach with the standard ones. Besides, Proposition 2 shows that the BLF-Pareto criterion and the BLF-biposs criterion are generalisation of the criteria maxmin and maxmax, indeed a decision based on an optimistic (respectively pessimistic) criteria can be computed by using Pareto or biposs criteria on a BLF containing only negative goals (respectively positive goals). Intuitively, moving goals from the positive side to the negative one amounts to move from considering that their existence is wished to considering that their absence is dreaded. The converse move that takes a goal from a negative side to a positive side is transforming something that is dreaded into something whose absence is wished. By balancing goals on the positive and negative sides, the decision maker may mitigate her pessimism. One special case is the $R_{*}^{e}$-BLF (see Def.11) with Pareto preference rules which capture the $R_{*}^{e}$ behavior.

Definition 11. A $R_{*}^{e}$-BLF is a fuzzy-BLF such that

- there is a universal positive goal $g^{u}$ and a DP: $\top \rightsquigarrow g^{u}$ with $l\left(g^{u}\right)>$ $\max _{g \in G \ominus l} l(g)$,

- for all $g$ in $G^{\oplus} \backslash\left\{g^{u}\right\}, \neg g \in G^{\ominus}$ and vice-versa,

- $\max _{g \in G \oplus} l(g)<e$,

- and if $l(g)>l\left(g^{\prime}\right)$ then $l(\neg g)>l\left(\neg g^{\prime}\right)$.

Proposition 3. An optimal solution using Pareto preference rules on a $R_{*}^{e}$-BLF is optimal for $R_{*}^{e}$ preference. 


\section{Discussion and concluding remarks}

We have shown that the BLF allows us to generalise the methods for solving qualitative decision problems under uncertainty because they were defined only on one scale. The extension to a bipolar scale is important to help to differentiate the ways to deal with the uncertainty associated with positive or negative goal, since their interpretation is not necessarily symmetric. The fact that the same problem may be represented with different polarities yields the main result that BLF generalizes the main classical qualitative decision approaches. Moreover, we have provided a formal characterization of the fact that BLF is a generalization of maxmin and maxmax criterion and we have situated it wrt to $R_{*}^{e}$-criterion.

The reader may wonder about the link with Cumulative Prospect Theory (CPT) of Tversky and Kahneman [14]. Admittedly, CPT only deals with decision making under risk (i.e. known probabilities), but it shares with BLF the distinction between positive and negative outcomes. More precisely, CPT starts with gains together with their probabilities and computes utilities according to risk adverse attitude. Hence it is a transformation of gain using a utility function. In contrast, our model thanks to the bipolarity, is dealing with utility and dis-utility with either pessimistic attitude (closely related to risk adverse attitude) or optimistic attitude.

\section{References}

[1] R Duncan Luce and Howard Raiffa, Games and decisions: Introduction and critical survey, Courier Corporation, 1989.

[2] John T Cacioppo and Gary G Berntson, "Relationship between attitudes and evaluative space: A critical review, with emphasis on the separability of positive and negative substrates.," Psychological bulletin, vol. 115, no. 3, pp. 401, 1994.

[3] S. Benferhat, D. Dubois, and H. Prade, "Representing default rules in possibilistic logic," in $K R, 1992$, pp. 673-684.

[4] Florence Dupin De Saint Cyr and Romain Guillaume, "Analyzing a Bipolar Decision Structure through Qualitative Decision Theory," KI - Künstliche Intelligenz, vol. 31, no. 1, pp. 53-62, mars 2017.

[5] Florence Dupin De Saint Cyr and Romain Guillaume, "How Potential BLFs Can Help to Decide under Incomplete Knowledge," in IPMU. 2018, Springer.

[6] Florence Dupin De Saint Cyr, Romain Guillaume, and Umer Mushtaq, "Explainable Decisions under Incomplete Knowledge with Supports and Weights," in FUZZ-IEEE, juin 2019, pp. 1-6. 
[7] Phan H Giang, "Decision making under uncertainty comprising complete ignorance and probability," International Journal of Approximate Reasoning, vol. 62, pp. 27-45, 2015.

[8] Hélène Fargier and Romain Guillaume, "Sequential decision making under ordinal uncertainty: A qualitative alternative to the Hurwicz criterion," IJAR, vol. 116, pp. 1-18, 2020.

[9] Salvatore Greco and Fabio Rindone, "Bipolar fuzzy integrals," Fuzzy Sets and Systems, vol. 220, pp. 21-33, 2013.

[10] Leonard J Savage, The foundations of statistics, Courier Corporation, 1972.

[11] Kenneth J Arrow and Leonid Hurwicz, "An optimality criterion for decision-making under ignorance," Uncertainty and expectations in economics, pp. 1-11, 1972.

[12] Hélène Fargier and Romain Guillaume, "Sequential decision making under uncertainty: Ordinal uninorms vs. the hurwicz criterion," in International Conference on Information Processing and Management of Uncertainty in Knowledge-Based Systems. Springer, 2018, pp. 578-590.

[13] JF. Bonnefon, D. Dubois, and H. Fargier, "An overview of bipolar qualitative decision rules," in Preferences and Similarities, vol 504, CISM, pp. 47-73. Springer, 2008.

[14] Amos Tversky and Daniel Kahneman, "Advances in prospect theory: Cumulative representation of uncertainty," Journal of Risk and uncertainty, vol. 5, no. 4, pp. 297-323, 1992. 